\title{
Impact of Brain Fatty Acid Signaling on Peripheral Insulin Action in Mice
}

\author{
Authors \\ Susanne Neschen ${ }^{1,2 *}$, Moya $\mathrm{Wu}^{1,2^{*}}$, Christiane Fuchs ${ }^{3,4^{*}}$, Ivan Kondofersky3, ${ }^{3,}$, Fabian J. Theis ${ }^{3,4}$, \\ Martin Hrabě de Angelis', 2, Hans-Ulrich Häring2, 5, 6, Tina Sartorius², 5, 6
}

\section{Affiliations}

1 Institute of Experimental Genetics, German Mouse Clinic, Helmholtz Center Munich, German Research Center for Environmental Health, Neuherberg, Germany

2 German Center for Diabetes Research (DZD), Neuherberg, Germany

3 Institute of Computational Biology, Helmholtz Center Munich, German Research Center for Environmental Health, Neuherberg, Germany

4 Center for Mathematics, Chair of Mathematical Modeling of Biological Systems, Technical University of Munich, Garching, Germany

5 Department of Internal Medicine, Division of Endocrinology, Diabetology, Vascular Disease, Nephrology and Clinical Chemistry, University of Tuebingen, Tuebingen, Germany

6 Institute for Diabetes Research and Metabolic Diseases of the Helmholtz Center Munich at the University of Tuebingen (IDM), Tuebingen, Germany

\section{Key words}

brain, electrocorticography, euglycemic-hyperinsulinemic clamps, fatty acids, palmitic acid, radiotelemetry

$\begin{array}{ll}\text { received } & 02.07 .2018 \\ \text { revised } & 01.08 .2018 \\ \text { accepted } & 10.09 .2018\end{array}$

\section{Bibliography}

DOI https://doi.org/10.1055/a-0735-9533

Published online: 5.11.2018

Exp Clin Endocrinol Diabetes 2020; 128: 20-29

(C) J. A. Barth Verlag in Georg Thieme Verlag KG Stuttgart New York

ISSN 0947-7349

\section{Correspondence}

Tina Sartorius, PhD

Department of Internal Medicine

Division of Endocrinology, Diabetology, Vascular Disease, Nephrology and Clinical Chemistry

University of Tuebingen

Otfried-Mueller-Str. 10,

72076 Tuebingen, Germany

sarto.ti@web.de
丹 Supporting Information Figure 1S-3S and Table 1S Online content viewable at https://doi.org/10.1055/a-0735-9533

\section{ABSTRACT}

Aims and Methods Glucose homeostasis and energy balance are under control by peripheral and brain processes. Especially insulin signaling in the brain seems to impact whole body glucose homeostasis and interacts with fatty acid signaling. In humans circulating saturated fatty acids are negatively associated with brain insulin action while animal studies suggest both positive and negative interactions of fatty acids and insulin brain action. This apparent discrepancy might reflect a difference between acute and chronic fatty acid signaling. To address this question we investigated the acute effect of an intracerebroventricular palmitic acid administration on peripheral glucose homeostasis. We developed and implemented a method for simultaneous monitoring of brain activity and peripheral insulin action in freely moving mice by combining radiotelemetry electrocorticography (ECoG) and euglycemic-hyperinsulinemic clamps. This method allowed gaining insight in the early kinetics of brain fatty acid signaling and its contemporaneous effect on liver function in vivo, which, to our knowledge, has not been assessed so far in mice.

Results Insulin-induced brain activity in the theta and beta band was decreased by acute intracerebroventricular application of palmitic acid. Peripherally it amplified insulin action as demonstrated by a significant inhibition of endogenous glucose production and increased glucose infusion rate. Moreover, our results further revealed that the brain effect of peripheral insulin is modulated by palmitic acid load in the brain.

Conclusion These findings suggest that insulin action is amplified in the periphery and attenuated in the brain by acute palmitic acid application. Thus, our results indicate that acute palmitic acid signaling in the brain may be different from chronic effects.

* These authors contributed equally to this work. 


$\begin{array}{ll}\text { ABBREVIATIONS } \\ \text { BG } & \text { blood glucose } \\ \text { CNS } & \text { central nervous system } \\ \text { CSF } & \text { cerebrospinal fluid } \\ \text { ECoG } & \text { electrocorticography } \\ \text { EndoRa } & \text { endogenous glucose production } \\ \text { GIR } & \text { glucose infusion rate } \\ \text { HFD } & \text { high fat diet } \\ \text { i.a. } & \text { intraarterial } \\ \text { i.c.v. } & \text { intracerebroventricular } \\ \text { LCFAs } & \text { long-chain fatty acids }\end{array}$

\section{Introduction}

Numerous studies in animals and humans have indicated that insulin action in the brain influences peripheral metabolism, body weight regulation, food intake and energy expenditure [1-4]. An intact insulin signaling chain in the brain is required for the full inhibitory effect of systemic insulin on endogenous glucose production $[5,6]$.

High caloric intake, high-fat feeding and overeating, common problems of Western societies, lead to insulin resistance in both the periphery and the central nervous system culminating in hyperglycemia and finally type 2 diabetes [7]. One of the earliest studies on insulin responses in the human brain revealed that, in obese humans, the brain is resistant to insulin as reflected by impaired cerebrocortical activity which was assessed by magnetoencephalographic measurements [8]. This attenuated brain response to insulin was also observed in subjects with genetically reduced insulin signaling at the level of the IRS-1 pathway [8] or in carriers of the FTO risk allele [9]. In obese and diabetic individuals elevated levels of circulating long-chain fatty acids (LCFAs) are prevalent and predominantly saturated free fatty acids have been suggested as causative link between obesity and insulin resistance in the liver and the brain $[10,11]$. Previously, we demonstrated that impaired insulin action in the human brain is associated with increased saturated nonesterified fatty acids (NEFAs) in the serum [10]. Cerebrocortical activity increased with insulin infusion during a hyperinsulinemic-euglycemic clamp in healthy subjects, and beta and theta activity were predominantly increased whereas overweight humans failed to respond to insulin [8]. However the underlying mechanisms cannot be easily studied in humans, therefore animal models are to be used. High-fat diet based on lard was shown to mediate insulin resistance in the mouse brain and impairs cortical activity and locomotion, which might further promote glucose intolerance, physical inactivity, and obesity [12].

There is evidence that Toll-like receptors (TLR) 2 and 4 [13] and fatty acid binding proteins are involved in SFA-induced signaling in nerve tissues with subsequent modulation of intracellular signaling pathways [14]. However, whether such chronically elevated levels of predominantly saturated NEFAs are causal for brain insulin resistance, or if impaired insulin action in the brain contributes to impaired glucose homeostasis and visceral fat accumulation in the long-term remains open.
Research in animals points towards an altered insulin action in the periphery by direct effects of different fatty acids in the brain. By central administration, short-term hypothalamic accumulation of lipid-derived metabolites, such as LCFAs, were shown to play an important role in lipid sensing of the brain and subsequent suppression of hepatic glucose production $[5,15,16]$. First evidence for an acute effect of brain-derived LCFAs on glucose homeostasis was demonstrated by a continuous intracerebroventricular oleic acid infusion lasting $6 \mathrm{~h}$ which lowered plasma insulin and glucose levels under basal physiological conditions in rats [16]. For this effect, activation of hypothalamic ATP-sensitive potassium ( $\left.\mathrm{K}_{\text {ATP }}\right)$ channels is necessary for insulin and lipids to lower hepatic glucose production via vagal input to the liver $[15,17]$. Regarding brainmediated inhibition of hepatic glucose production, LCFAs were shown to differentially impair systemic insulin action depending on the degrees of unsaturation [18-20], and diet enrichment with monounsaturated contrary to saturated fatty acids protected from impairments in cerebrocortical activity and brain insulin sensitivity $[12,20]$. Of note, it is suggested that even hyperinsulinemia in the presence of hyperlipidemia may impair lipid-sensing mechanisms in the brain as demonstrated by hyperinsulinemic-euglycemic clamp experimental models independent of obesity or dietinduced hyperinsulinemia [21-24]. Furthermore, an intestinebrain-liver neural axis was shown to be implicated in the regulation of hepatic glucose production by upper intestinal lipids, and hepatic vagotomy abolished their inhibitory effects on glucose production [25]. However, although the liver and the brain share molecular pathways, one has to mention that opposing physiological mechanisms are assumed to balance glucose homeostasis [26] as accumulation of LCFAs in the liver reduced liver insulin action and increased glucose production [27].

Abovementioned studies gained deep insight into a hypothalamic and intestinal lipid sensing in modulating liver glucose homeostasis through a neural network [15-17, 25, 28]; however, these diet- or fatty acids-induced metabolic effects did not reflect contemporaneous kinetics. Although previous studies revealed that hypothalamic accumulation of LCFA-CoAs, either by intracerebroventricular (i.c.v.)/ intrahypothalamic (i.h.) administered fatty acids [16,29] or by systemic Intralipid infusion [15], led to suppression of hepatic glucose production, the design of the experiments was based on continuous infusions lasting 4 (i. v.) or $6 \mathrm{~h}$ (i.c.v./ i.h.). Therefore, it remains to be elucidated if such peripheral effects on glucose homeostasis are a consequence of the acute fatty acid signaling brain or due to secondary fatty acid-induced brain alterations like inflammation. Hypothalamic inflammation is induced within a few hours after HFD-onset and may temporarily subside due to neuroprotective mechanisms [30]. In this regard, an increase of proinflammatory genes such as $1 / 6, T n f \alpha$, and Socs 3 was detectable within the first $72 \mathrm{~h}$ of HFD exposure, but transiently declined to baseline values until day 14 due to neuroprotective microglial and astroglial responses (accumulation and enlargement) as consequence of the initial insult directed at hypothalamic neurons. This indicates that inflammatory processes in the brain, especially in the hypothalamus, occur quite rapidly after a fatty acid challenge and may be independent of systemic inflammation occurring on a longer time scale. 
Thus, the aim of the current study was to develop a method which allows to assess simultaneously the early kinetics of brain fatty acid signaling as well as the effect on liver function in vivo. For this reason, we designed the current study to (i) provide a method by which cerebrocortical activity and glucose homeostasis could be simultaneously assessed in freely moving mice, (ii) explore whether and to what extent centrally applied fatty acids, especially palmitic acid, simultaneously impact brain activity and hepatic glucose production under hyperinsulinemic-euglycemic conditions, and (iii) reveal if contemporaneous, reciprocal changes in hepatic gluconeogenesis and glycogenolysis compensate for cerebrocortical activity variations when CSF palmitic acid concentrations and systemic insulin levels were increased.

\section{Materials and Methods}

\section{Animals}

C57BL/6NTac (Taconic, Ejby Lille Skensved, Denmark) mice were bred and housed in a temperature- and humidity-controlled environment (12:12 light:dark-cycle) in individually ventilated cages (Tecniplast, Hohenpeißenberg, Germany) and in compliance with Federation of European Laboratory Animal Science Associations protocols. All animals had free access to water and a mouse laboratory chow (Diet\#1310, Altromin, Lage, Germany). After weaning, male mice were housed in groups. Surgeries were conducted in 12-14 weeks old mice which were thereafter individually housed in cages containing nesting material and dome houses. All procedures were performed according to the guidelines of laboratory animal care and approved by the Upper-Bavarian district government (AZ 55.2-1-54-2532-153-12).

\section{Surgical procedures}

Permanent catheterisation of jugular vein and carotid artery

Catheter preparation A permanent catheter, prepared from two different tubing, was implanted in the carotid artery for blood sampling in freely moving mice ( $\vee$ Fig. 1a, 1Sa). A polyethylene tubing (PE-10, I.D. $0.28 \mathrm{~mm}$, O.D. $0.61 \mathrm{~mm}$, Intramedic, Becton Dickinson, New Jersey, USA) was carefully stretched to reduce its diameter by approximately $40 \%$, and the tip was inserted into a $100 \mathrm{~mm}$ piece of silicon tubing (I.D. $0.30 \mathrm{~mm}$, O.D. $0.60 \mathrm{~mm}$, Sani Tech, Ohio, USA). A $1 \mathrm{~mm}$ 'sleeve' prepared from silastic tubing (I.D. $0.51 \mathrm{~mm}$, O.D. $0.94 \mathrm{~mm}$, Dow Corning, Michigan, USA) was stretched with a delicate forceps (RS-5047, Dumont, Montignez, Switzerland) and placed over the overlapping catheter area to smoothen and stabilize it. During surgery, the catheter was inserted $9 \mathrm{~mm}$ into the carotid artery until sleeve-touching, thereby placing its tip in the entrance area of the aortic arch. The jugular vein catheter ( $\triangleright$ Fig. 1a, 1Sa) used for substrate infusion consisted of a $110 \mathrm{~mm}$ long silicon tubing (I.D. $0.30 \mathrm{~mm}$, O.D. $0.60 \mathrm{~mm}$, Sani Tech, Ohio, USA) introduced $11 \mathrm{~mm}$ into the blood vessel. The position was marked with liquid silicone, applied like a sleeve around the tubing. Both catheter tips were cut blunt with a scalpel to the right length.

Surgical catheterization Surgical catheterization was performed $7 \pm 2$ days prior to in vivo experiments. Anaesthesia was provided by an intraperitoneally applied cocktail of Medetomidin $(0.5 \mathrm{mg} / \mathrm{kg}$ Domitor ${ }^{\circledR}$ ), Midazolam (5 mg/kg Midazolamratiopharm ${ }^{\circledR}$ Dormicum) and Fentanyl $\left(0.05 \mathrm{mg} / \mathrm{kg}\right.$ Fentanyl ${ }^{\circledR}$ Janssen WdT). Hair was removed from a ventral area above the sternum and from the dorsal, interscapular surgical site. A vertical midline incision was made in the skin above the mandibular glands. After gentle separation of the omohyoid from the sternohyoid muscle, the left carotid was exposed by carefully detaching the adjacent vagal nerve. Cranial the artery was irreversibly ligated (braided silk suture 6-0, Teleflex, Conventry, USA) and a vascular clamp (S\&T B-1 Vascular clamp, F.S.T. Heidelberg, Germany) was placed at the most distal posterior position possible to temporarily stop the blood flow.

After suture material was loosely tied around the exposed carotid, the blood vessel was punctured with 27 -gauge needle. The PE-10 catheter tip, prefilled with heparinized saline $(200 \mathrm{lU} / \mathrm{mL})$, was inserted by enlarging the puncture hole, the vascular clamp was released and the catheter advanced until the silicone sleeve. The catheter was fixed in position by three ligations.

For jugular cannulation a ventral skin incision approximately $5 \mathrm{~mm}$ to the right of the midline cephalic to the sternum was made and the external right jugular vein was exposed. Cranial the vein was irreversibly ligated with braided silk suture (6-0, Teleflex, Conventry, USA), punctured, and the catheter introduced and fixed in position with ligatures. To confirm their patency catheters were flushed with heparinized saline. The carotid catheter was filled with Taurolock solution (TauroPharm GmbH, Waldbüttelbrunn, Germany). Both catheter ends were sealed with tight knots. An interscapular skin incision enabled to subcutaneously tunnel catheter ends from ventral to dorsal. Catheters were stowed in a subcutaneous skin pocket during post-surgical recovery, whereat one strand of sterile surgical silk (Terylene 5-0, Serag, Naila, Germany) was tied to each silicone catheter end with a knot and remained partially exposed to recover the catheter ends prior to infusion. Ventral and dorsal incisions were closed with sutures (Perma-Hand Seide 5-0, Ethicon, Norderstedt, Germany). Silk ends were cut short ( 10 mm) and soaked with Betaisodona solution. An intraperitoneal injection of Atipamezol (2.5 mg/kg, Antisedan $\left.{ }^{\circledR}\right)$, Flumazenil $(0.5 \mathrm{mg} / \mathrm{kg}$, Anexate ${ }^{\circledR}$ Hexal), and Naloxon $\left(1.2 \mathrm{mg} / \mathrm{kg}\right.$, Naloxon $\left.{ }^{\circledR}\right)$ antagonized anesthesia.

Intracerebroventricular (i.c.v.) cannulation and implantation of electrocorticography (ECoG) radiotelemetry transmitters

In the second surgery ( $\triangleright$ Fig. 1b) an ECoG transmitter ( $\triangleright$ Fig. 1Sb) and an intracerebroventricular (i.c.v.) cannula for substrate injection into the CSF ( $\triangleright$ Fig. 1Sc) were installed as follows: One day after catheterization mice were anaesthetized with isoflurane $(1.5 \%$; mixed with $4 \mathrm{~L} /$ min oxygen) for insertion of radiotelemetric implants and an i.c.v. cannula. Body temperature was maintained at $37^{\circ} \mathrm{C}$, and anesthesia depth was determined by respiration rate, vibrissae movements, and eyelid reflex. A stereotaxic head holder enabled subcutaneous implantation of the telemetry ECoG transmitter (TA-F20, Data Sciences International, Minnesota, USA) in anaesthetized mice as previously published [20] ( ECoG lead wires were connected to screws placed epidurally $(1 \mathrm{~mm}$ anterior to lambda and $1 \mathrm{~mm}$ left to the sutura sagittalis; $1 \mathrm{~mm}$ anterior to bregma and $1 \mathrm{~mm}$ right to the sutura coronaria). An i.c.v. 


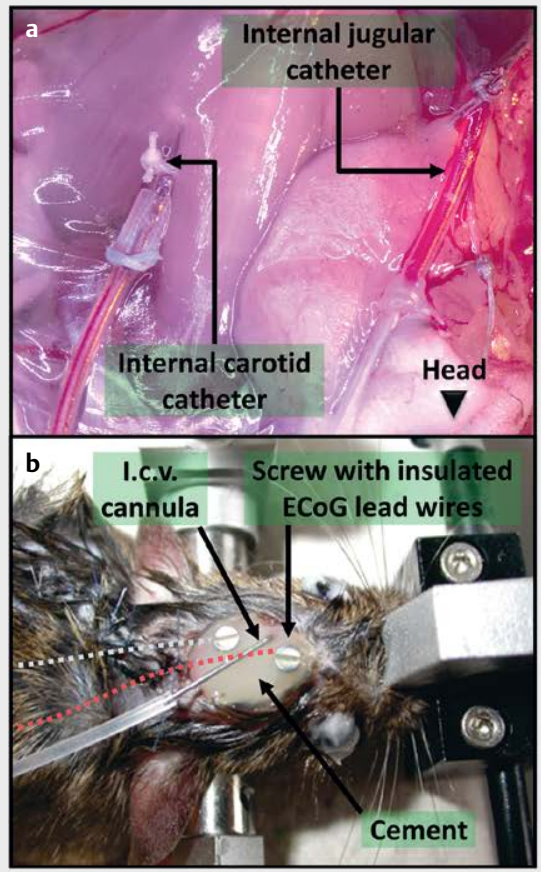

\section{d}

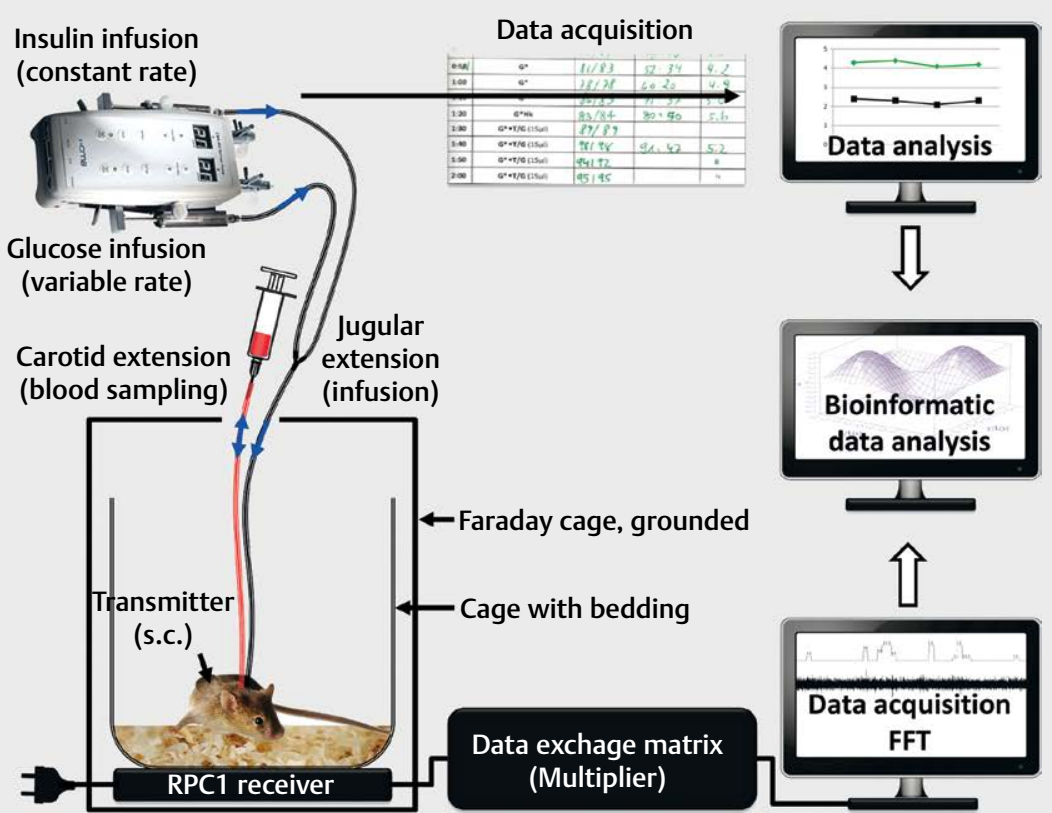

C

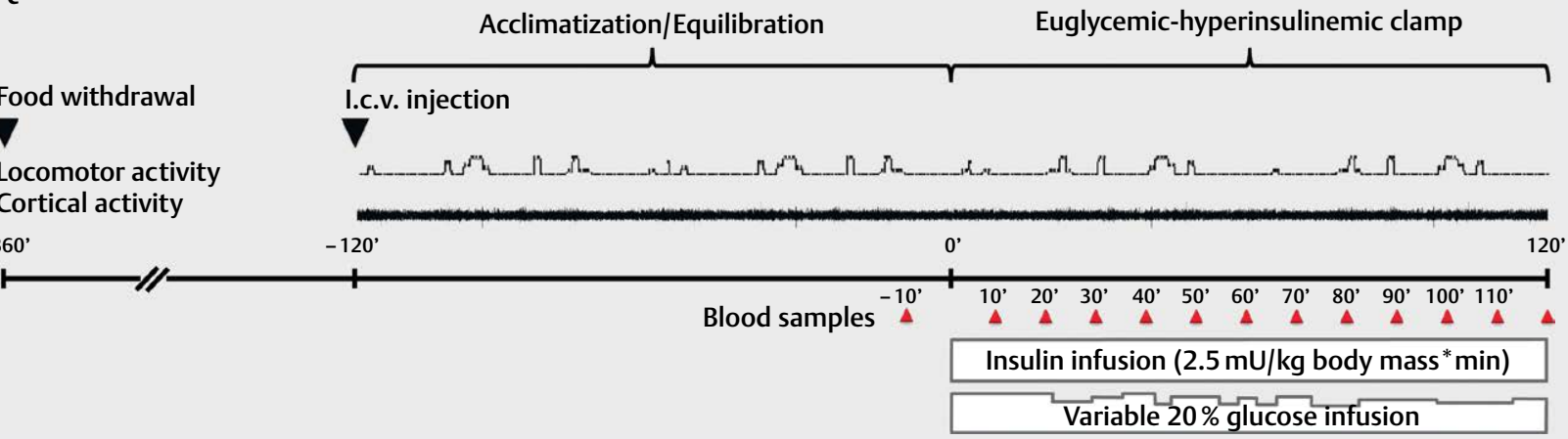

- Fig. 1 Synchronous assessment of cerebrocortical activity and insulin action in freely moving mice. a Installed silicone catheter inserted into the right jugular vein and silicone-polyethylene catheter introduced into the left carotid artery after careful vagal nerve separation. b Each indicated wire (red and white dashed lines) connects an epidural screw with the telemetry electrocorticography transmitter. c,d Depiction of the time line $\mathbf{c}$ and setup $\mathbf{d}$ for the simultaneous assessment of brain activity and hepatic glucose homeostasis. During the clamp and simultaneous radiotelemetry recordings, blood samples are taken every $10 \mathrm{~min}$ to measure blood glucose. The glucose infusion rate is adjusted accordingly to maintain euglycemia. Baseline samples for blood glucose, plasma insulin, and plasma $\left[3-{ }^{-3} \mathrm{H}\right]$-glucose are taken at $\mathrm{t}=-10$ and $0 \mathrm{~min}$.

cannula (27-G, $7.5 \mathrm{~mm}$ in length, B/Braun, Melsungen, Germany) ( $\triangleright$ Fig. 1Sc) was additionally implanted in the left ventricle $(0.3 \mathrm{~mm}$ posterior and $1 \mathrm{~mm}$ lateral relative to bregma, and $3.0 \mathrm{~mm}$ down the skull surface) to directly inject substances in the cerebrospinal fluid. Electrodes and screws were fixed in place with dental acrylic cement, whereat special care was taken to avoid entwining catheters and transmitter wires.

\section{Post-operative medication and health monitoring}

For pre-emptive analgesia Carprofen (Rimadyl ${ }^{\circledR}, 5 \mathrm{mg} / \mathrm{kg}$ ) was subcutaneously administered and further provided once daily for the consecutive two post-surgical days. The recovery of each animal was carefully monitored using specific score sheets that defined experimental endpoints ( $\triangleright$ Table 1S).

\section{Fatty acids}

A vehicle solution was prepared from absolute ethanol and $\mathrm{KRH}$ buffer ( $50 \mathrm{mM}$ HEPES, $137 \mathrm{mM} \mathrm{NaCl}, 4.8 \mathrm{mM} \mathrm{KCl}, 1.85 \mathrm{mM} \mathrm{CaCl}_{2}$, $1.3 \mathrm{mM} \mathrm{MgSO}_{4}, \mathrm{pH} 7.4$ ) supplemented with fatty acid-free BSA ( $75 \mu \mathrm{M}$, Serva, Heidelberg, Germany). In this solution, palmitic acid (C16:0), oleic acid (C18:1), or linoleic acid (C18:2; all from Cayman Chemical, Michigan, USA) were solubilized ( $2.92 \mathrm{mM}$ final concentration). Thus, by infusing $5 \mu$ in the cerebrospinal fluid of the mouse, a final concentration of $225 \mu \mathrm{M}$ (containing $0.11 \% \mathrm{EtOH}$ ) was achieved.

\section{I.c.v. application and radiotelemetric measurements}

Mice recovered 8 days from surgeries. To acclimatize mice to the experimental environment each individual was transferred to a 
plastic container (diameter $170 \mathrm{~mm}$ ) with home-cage bedding at 6 a.m. ( $t=-360 \mathrm{~min}$ ) with no access to food but free access to water ( $\triangleright$ Fig. 1d). After $4 \mathrm{~h}$ food deprivation ( $\mathrm{t}=-120 \mathrm{~min}$ ) a volume of $5 \mu$ L vehicle $(n=8)$ or fatty acid solution ( $n=6-8 /$ group) was delivered at a flow rate of $2 \mu \mathrm{L} / \mathrm{min}$. The infusion was performed by connecting the cannula via a polyethylene catheter to a microperfusion pump (CMA/Microdialysis, Solna, Sweden). Telemetry signals (ECoG and locomotion) were recorded continuously until the end of the experiment $(t=120 \mathrm{~min})$.

\section{Euglycemic hyperinsulinemic clamps}

Catheter ends were recovered, opened, and the fluid in the catheters was gently aspirated and discarded. The catheters were flushed with a sterile heparinized saline (10 U heparin/mL saline) solution and connected to micro-renathane tubing (Braintree Scientific, Massachusetts, USA) using a fine dosage syringe (Omnifix F, Braun, Melsungen, Germany). The venous catheter was used for substrate infusion performed with a microperfusion pump (CMA, Microdialysis, Solna, Sweden). The carotid catheter was used to withdraw blood and clamped between blood sampling to avoid reflux. Withdrawn blood volume was $50 \mu \mathrm{L}$ at $\mathrm{t}=10 \mathrm{~min}$, and $25 \mu \mathrm{L}$ at $\mathrm{t}=90,100,110$, and $120 \mathrm{~min}$. Blood volume was calculated with hematocrit $=40 \%$. The equilibration period of $120 \mathrm{~min}(\mathrm{t}=-120 \mathrm{~min}$, prior to insulin infusion) was started with injecting a $37 \mathrm{kBq}\left[3-{ }^{3} \mathrm{H}\right]$-glucose bolus via the jugular vein catheter followed by a continuous infusion of $\left[3-{ }^{3} \mathrm{H}\right]$ glucose at a rate of $1.85 \mathrm{kBq} / \mathrm{min}$. At minute $\mathrm{t}=-10$ an arterial blood sample for basal plasma glucose, $\left[3-{ }^{3} \mathrm{H}\right]$ glucose and insulin measurements was collected in potassium-EDTA-coated tubes (Microvette CB300, Sarstedt, Nürnbrecht, Germany). At $t=0$ min, the start of the euglycemic-hyperinsulinemic clamp, insulin (15 pmol/ $\mathrm{kg}^{*} \mathrm{~min}^{-1}$; HumulinR, Lilly, USA) and [3-3 $\mathrm{H}]$-glucose $(3.7 \mathrm{kBq} / \mathrm{min})$ were infused via the jugular vein catheter. Glucose concentrations in arterial blood were measured every 10 min (Bayer Contour, Germany) and blood glucose fluctuations adjusted by varying the rate of a $20 \%$ glucose solution (Glucose Infusion Rate, GIR). After $80 \mathrm{~min}$ ( $\mathrm{t}=80 \mathrm{~min}$ ) additional blood was collected, plasma harvested and further used for determination of plasma tracer radioactivity, insulin (mouse insulin ELISA, Mercodia, Uppsala, Sweden), and glucose (LabAssay ${ }^{\mathrm{TW}}$ Glucose, Wako Chemicals, Richmond, USA) concentration measurements. All infusions were performed with microperfusion pumps (CMA402, Solna, Sweden) and radioisotopes were purchased from Perkin Elmer (Boston, USA). At the end of experiments animals were killed with i.a. ketamine/xylazine.

\section{ECoG data pre-processing}

Pre-processing of ECoG measurements was performed using fast Fourier transformation (FFT) for different frequency bands (theta $(4-8 \mathrm{~Hz})$, beta $(12-30 \mathrm{~Hz}))$ to calculate the power spectral density in $\left(\mu \mathrm{V}^{2} / \mathrm{Hz}\right)$. Inter-individual variation in baseline cortical activity was excluded by expressing the data for the ECoG power density as percentage change from baseline $\left(\left[3-{ }^{3} \mathrm{H}\right]\right.$ glucose bolus $(0.05 \mu \mathrm{Ci})$ min) lasting 120 min with $0 \%$ as baseline. Data are presented as mean \pm SEM for the injection period with $\left[3-{ }^{3} \mathrm{H}\right]$ glucose bolus $(0.1 \mu \mathrm{Ci} / \mathrm{min})$; insulin $(2.5 \mathrm{mU} / \mathrm{kg} / \mathrm{min})$ and a variable $20 \%$ glucose infusion lasting $120 \mathrm{~min}$. ECoG signals were acquired digitally by using Somnologica Science software (version 3.3.1; Medcare, Reykjavík, Iceland) and NeuroScore software (version 3.0; DSI).

\section{Statistical analysis for ECoG data and parameters of glucose homeostasis}

For detecting differences in time courses of ECoG and glucose homeostasis parameters (endogenous glucose production (EndoRa), blood glucose (BG), glucose infusion rate (GIR)) a functional test was employed [31]. Two-dimensional Pearson correlation patterns were applied for every pair of parameters and within each of the four treatment and vehicle groups. Frequency bands were available at intervals of two seconds over the euglycemic-hyperinsulinemic clamp time span of $120 \mathrm{~min}$, and the last $40 \mathrm{~min}(\mathrm{t}=80-$ $120 \mathrm{~min}$ ) were defined as steady state. Due to computational analysis effort, frequency band measurements were aggregated by local averaging such that time series contained no more than 500 measurements. Such aggregation may cause higher correlations than one would observe in non-aggregated data [32]. Only differences between groups and not absolute values of the correlations were investigated. Having calculated correlation patterns for two groups, differences between the two matrices were calculated and summed up over all absolute values of the resulting matrix. This yielded our test statistic. For permutation testing mice were randomly assigned to any of the two considered groups for 500 times, and the above calculation of the test statistic was repeated for every such assignment.

\section{Results}

We first established the combination of two highly complex techniques - radiotelemetry ECoG and the glucose clamp technique - to simultaneously assess brain activity and whole body- and organ-specific insulin action in freely moving mice ( $\mathbf{F i g}$. 1d). Surgeries were performed on two consecutive days to substantially enhance the survival rate and post-surgical recovery of mice with a body mass $>25 \mathrm{~g}$. Each animal received extensive post-surgical care and its recovery was scored daily ( $\triangleright$ Table 15$)$. Following a decrease in body mass in the first two post-surgical days $(9.5 \pm 1.1 \%$ body mass change from pre-surgical body mass), $90 \%$ of mice increased and almost completely restored their pre-surgical body mass ( $\triangleright$ Fig. 2Sa).

In vivo experiments were conducted eight days after the first surgery ( $\triangleright$ Fig. 1c) as elongation of the post-surgical recovery period limited the functionality of the catheters and i.c.v. cannula.

\section{VIDEO}

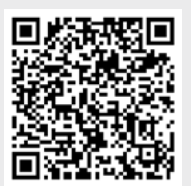

https://doi.org/

10.1055/a-0735-9533

- Video. 1 C57BL/6NTac mouse with permanent catheterisation of jugular vein and carotid artery, intracerebroventricular (i.c.v.) cannula and implanted radiotelemetry transmitter for electrocorticography $(\mathrm{ECOG})$ measurements. The mouse, located in a Faraday cage, well tolerates the combination of ECoG recordings and euglycemichyperinsulinemic clamps and is able to move freely and without restriction of its normal physical behavior. 

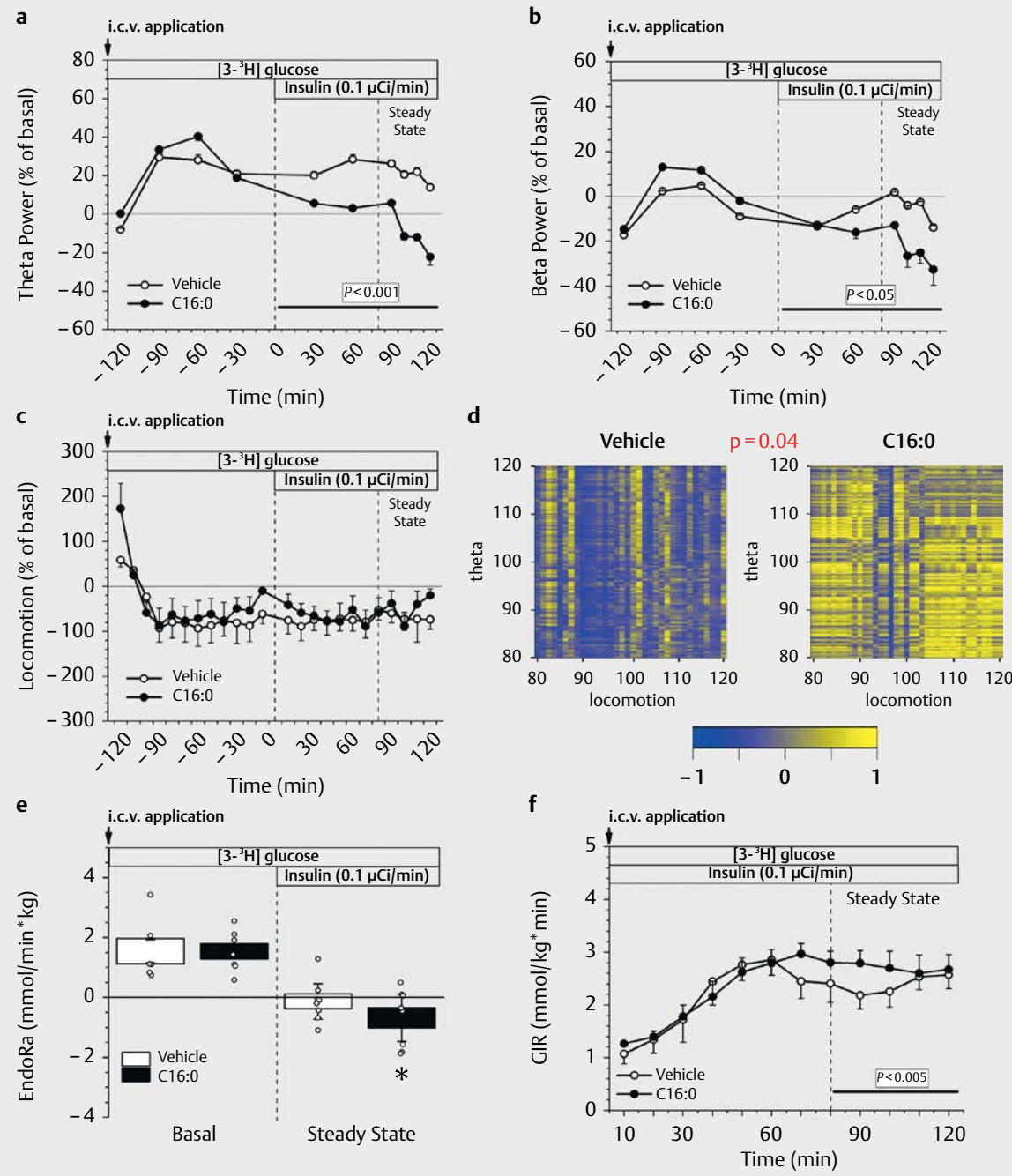

\begin{abstract}
- Fig. 2 Cerebrocortical activity, locomotion, endogenous glucose production (EndoRa) and glucose infusion rate (GIR) modulated by acutely intracerebroventricularly (i.c.v.) injected palmitic acid. Solutions of palmitic acid (C16:0) or vehicle were i.c.v. injected and theta $\mathbf{a}$ and beta $\mathbf{b}$ frequencies, locomotion $\mathbf{c}$, EndoRa $\mathbf{e}$ and GIR $\mathbf{f}$ were assessed during the equilibration period ( $\mathrm{t}=-120 \mathrm{~min}$, prior to insulin infusion with a continuous infusion of $\left[3-{ }^{-} \mathrm{H}\right]$-glucose) (cerebrocortical activity and locomotion) and the euglycemic-hyperinsulinemic clamp (cerebrocortical activity, EndoRa, GIR). Data are mean \pm SEM of $n=8 /$ group; data of each group are averaged over an interval of $30 \mathrm{~min} ;{ }^{*} \mathrm{P}<0.05$. $\mathbf{d} 2 \mathrm{D}$ time-resolved Pearson correlation patterns for theta vs. locomotion comparing vehicle against C16:0. P-value indicates significant pairwise differences between correlation patterns for these groups, and was calculated using permutation tests.
\end{abstract}

Four-hour fasted mice were transferred to a Faraday cage when te-

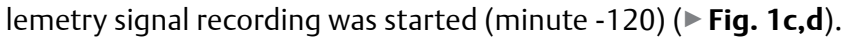
Radiotelemetric ECoG recordings were continuously assessed and in parallel, $6 \mathrm{~h}$ after food withdrawal, a 120 min-lasting euglycemichyperinsulinemic clamp was performed using a constant insulin infusion ( $2.5 \mathrm{mU} / \mathrm{kg}$ body mass * min) and maintaining euglycemia by a variable $20 \%$ glucose infusion ( $\vee$ Fig. 1 c,d). The mice well tolerated the combination of ECoG recordings and euglycemic-hyperinsulinemic clamps and were able to move freely and without restriction of their normal physical behavior (Video 1).

To examine whether co-signaling of specific fatty acids in the brain influences brain activity and simultaneously alters liver glucose homeostasis under hyperinsulinemic-euglycemic clamp conditions, we randomized four experimental groups. Lean, healthy
C57BL/6NTac male mice were intracerebroventricularly injected either with a saturated (palmitic acid, C16:0), monounsaturated (oleic acid, C18:1), or polyunsaturated (linoleic acid, C18:2) fatty acid or vehicle solution. I.c.v. application per se initially caused an increase of cerebrocortical activity independent of the fatty acids ( $\vee$ Fig. 2a,b, 3a,b), whereas palmitic acid elicited the most pronounced acute effect on cerebrocortical activity and caused a significant decrease of theta ( $>$ Fig. 2a) and beta frequency ( $>$ Fig. 2b) under hyperinsulinemic clamp-conditions predominantly in the steady state ( $\mathrm{t}=80-120 \mathrm{~min}$ ). Blood glucose (BG) levels conformed euglycemic conditions and were kept constant and near basal levels in all groups ( $>$ Fig. 2 Sb and $\triangleright$ Fig. $\mathbf{3 d}$ ).

We next investigated whether insulin-mediated locomotion, as previously demonstrated by i.c.v. applied insulin $[12,20]$, was af- 
a
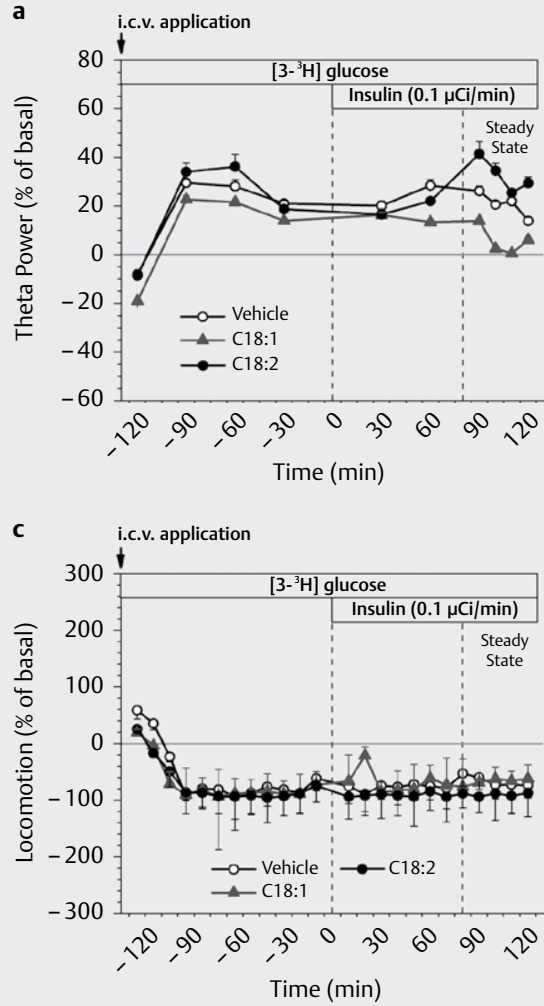

e

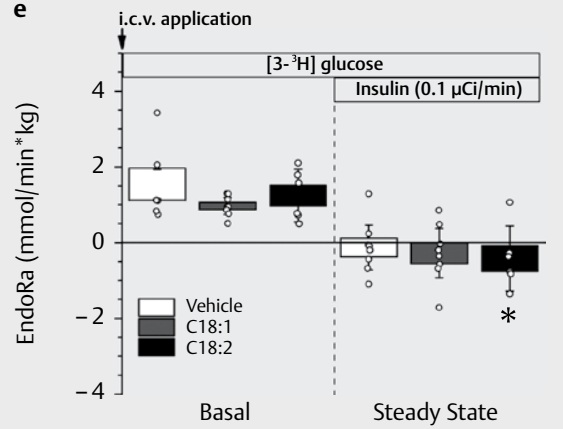

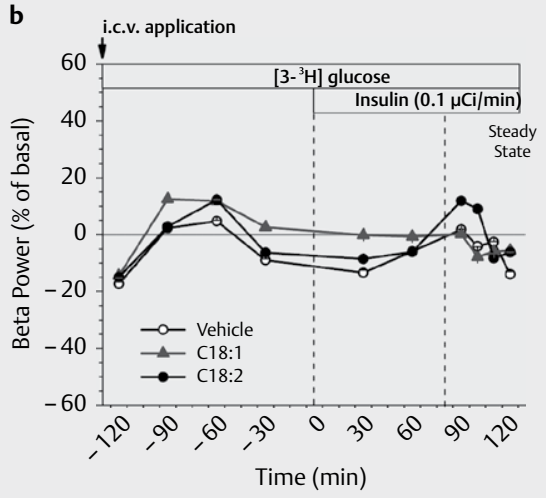

d
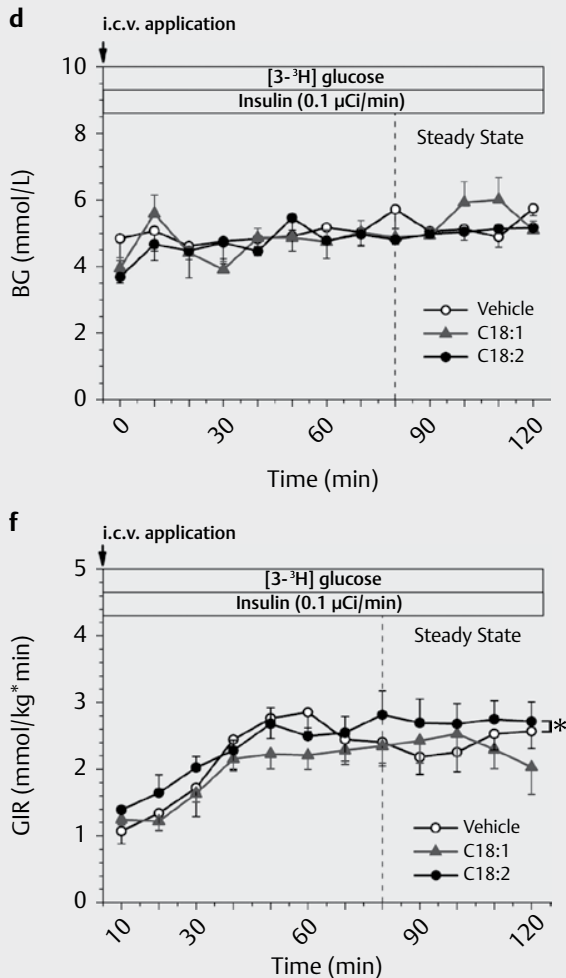

- Fig. 3 Cerebrocortical activity, locomotion, endogenous glucose production (EndoRa) and glucose infusion rate (GIR) modulated by acutely intracerebroventricularly (i.c.v.) injected oleic and linoleic acid. Solutions of oleic acid (C18:1), linoleic acid (C18:2), or vehicle were i.c.v. injected and theta $\mathbf{a}$ and beta $\mathbf{b}$ frequencies, locomotion $\mathbf{c}$, blood glucose (BG) d, EndoRa $\mathbf{e}$ and GIR $\mathbf{f}$ were assessed during the equilibration period ( $t=-120 \mathrm{~min}$, prior to insulin infusion with a continuous infusion of $\left[3-{ }^{3} \mathrm{H}\right]$-glucose) (cerebrocortical activity and locomotion) and the euglycemic-hyperinsulinemic clamp (cerebrocortical activity, BG, EndoRa, GIR). Data are mean \pm SEM of $n=6-8 /$ group; ${ }^{*} \mathrm{P}<0.05$.

fected by fatty acid co-signaling in the brain. By comparing separate time points during the clamp studies, neither i.c.v. palmitic acid ( $>$ Fig. 2c, 3Sa) nor oleic or linoleic acid ( $>$ Fig. 3c, 3Sb) had any significant effect on locomotion, either during hyperinsulinemic clamp conditions. This was further clarified by univariate analysis of time-resolved differences at $95 \%$ confidence level (grey shaded area in $\mathbf{F i g .} \mathbf{3 S a , b}$ ) between the respective groups. As the confidence band does cover the zero line (equates to functional mean difference; solid line in \ Fig. 3Sa,b), no significant difference resulted for the parameter locomotion or group comparison.

Of note, despite the marked decrease of brain activity in the theta and beta frequency bands during hyperinsulinemic-euglyce- mic clamp conditions, systemic insulin infusions led to a reduction in endogenous glucose production (EndoRa) in the presence of palmitic acid co-signaling in the brain ( $>$ Fig. 2e). Palmitic acid (C16:0) resulted in the most distinct inhibition of EndoRa compared to both unsaturated fatty acid species and vehicle ( $>$ Fig. $\mathbf{3 e}$ ), and glucose infusion rate (GIR) was significantly elevated in the steady state by C16:0 ( Fig. 2f) and C18:2 ( Fig. 3f), whereas oleic acid did not show any difference to vehicle ( $\triangleright$ Fig. $3 \mathbf{3 f})$. This amplification of subsequent peripheral insulin infusion indicates a higher whole-body insulin sensitivity which might contemporarily counteract acutely impaired brain activity. 
a

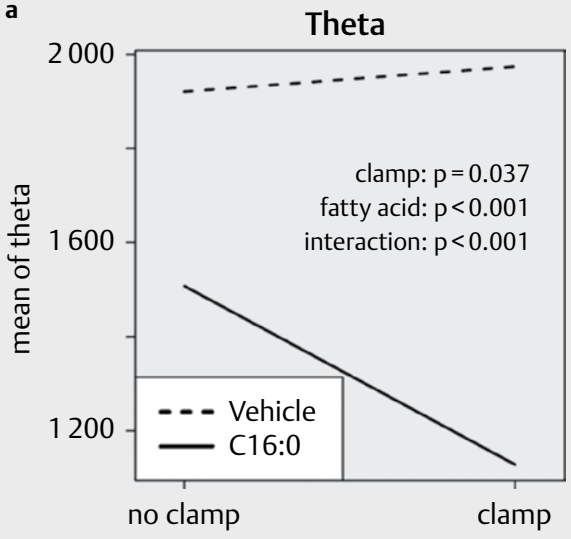

b

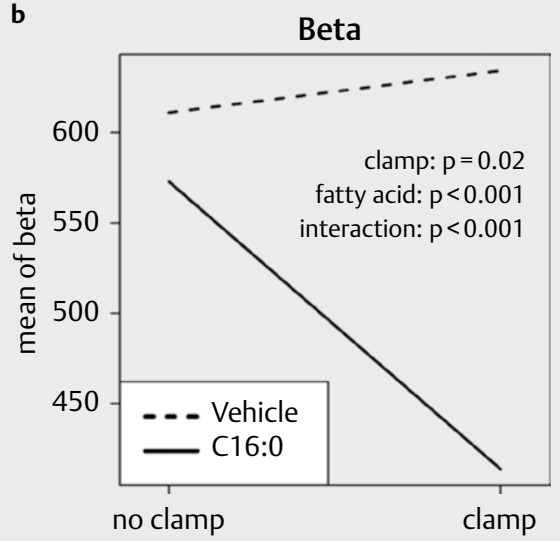

- Fig. 4 Interaction plots of cerebrocortical activity. $\mathbf{a}, \mathbf{b}$ Interaction plots for brain activity parameters theta $\mathbf{a}$ and beta $\mathbf{b}$, comparing mice before/ during the glucose clamp and with/without i.c.v. applied palmitic acid (C16:0). P-values result from a two-way ANOVA and show significant effects on frequency bands in terms of clamp, fatty acid and their interaction.

To further assess the individual and integrated effects of the two experimental interventions - the simultaneous assessment of cerebrocortical activity and whole body insulin action in freely moving mice - we investigated each experimental parameter in a twoway ANOVA, comparing mice before/during the glucose clamp and with/without i.c.v. applied fatty acids. As expected interaction plots revealed an increase of theta ( $\triangleright$ Fig. $4 \mathbf{4 a}$ ) and beta ( $\triangleright$ Fig. 4 b) frequencies in the vehicle group through systemic insulin infusion (clamp) compared to no-clamp condition, represented by a positive rise. Most pronounced effects were apparent between vehicle and C16:0 in all frequency bands of cerebrocortical activity. We found significance for theta ( $>$ Fig. $4 \mathbf{4 a}$ ) and beta ( $>$ Fig. 4b) frequencies in terms of clamp, fatty acid and interaction. Notably, in all cases, C16:0 caused a significantly decreased brain activity as compared to vehicle, and this impairment persisted significantly during the simultaneous glucose clamp and even further deteriorated brain activity.

\section{Discussion}

The present study provides new insight into a simultaneous effect of short-time accumulation of different fatty acid species on brain activity and hepatic glucose production in mice by using combined radiotelemetry and hyperinsulinemic-euglycemic clamp approaches. This is, to our knowledge, the first study revealing the time response of different intracerebroventricularly applied fatty acid species on brain activity and its contemporaneous effect on glucose homeostasis under hyperinsulinemia. Here, we showed that palmitate co-signaling in the brain is sufficient to attenuate brain activity in both theta and beta frequencies but amplified insulin action in the periphery as demonstrated by a significant inhibition of endogenous glucose production and increased glucose infusion rate. These results may indicate a contemporaneous counter-regulation of the liver in response to attenuated brain activity, and emphasize the brain as regulator of palmitate-induced modulation of hepatic glucose production. In this regard, previous work demonstrated that hypothalamic lipid sensing modulates liver glucose homeostasis through a neural network [15, 16, 28]. For instance, i.c.v. applied oleic acid lasting $6 \mathrm{~h}$ resulted in a lower glucose production and food intake in rodents [16], and intravenously infused Intralipid, a mixture of different fatty acid species of different degrees of unsaturation, was shown to regulate hepatic glucose production through hypothalamic signaling [15]. However, the conclusions of these studies are related to an infusion for hours and did not reflect contemporaneous kinetics revealing the time response of short-term brain accumulation of different fatty acid species. This is of importance as e.g. hypothalamic inflammation is induced within a few hours after HFD-onset and may temporarily subside due to neuroprotective mechanisms [30].

Further, it is to acknowledge that in light of our findings, hyperinsulinemic clamp conditions have a pivotal role in simultaneously modulating peripheral insulin action by palmitate co-signaling in the brain. Our results suggest that increased systemic insulin concentrations are necessary in parallel to palmitate co-signaling in the brain to attenuate brain activity in the theta and beta frequencies on the one hand and, remarkably, to amplify peripheral insulin action on the other hand.

Future experiments are necessary to characterize and elucidate the molecular signaling mechanisms underlying the insulin-amplified effect on hepatic glucose production when brain activity is simultaneously attenuated by palmitate co-signaling in the brain. However, with regard to palmitic acid, one might assume that palmitic acid elevates the intracellular ceramide content by increasing rates of de novo ceramide synthesis, potentially via TLR4 activation [33], that inhibit AKT and activates NFKB signaling [34, 35], thus influencing hepatic glucose production in our study. Moreover, isoforms of protein kinase $C$ (PKC- $\delta$, PKD- $\theta$, and PKC- $\varepsilon$ ) might be further activated which subsequently phosphorylate and activate ATPsensitive potassium ( $\mathrm{K}_{\text {ATP }}$ ) channels as LCFAs-CoA and the lipidderived metabolite diacylglycerol were shown to activate PKC isoforms [36, 37]. Of note, activation of $\mathrm{K}_{\text {ATP }}$ channels in the hypothalamus by drug infusion for hours was shown to be sufficient and obligatory for lipid-sensing in the brain to inhibit glucose production 
$[15,17]$. Regarding the impaired brain activity by palmitate cosignaling in the present study, one might speculate about an activation of $\mathrm{K}_{\mathrm{ATP}}$ channels in the brain which induces membrane hyperpolarization and therefore reduces the firing rate of neurons. This effect was more apparent when systemic insulin concentrations were increased during clamp conditions resulting in an additional attenuation of brain activity. As insulin was shown to equally activate $\mathrm{K}_{\text {ATP }}$ channels in hypothalamic neurons in vitro and in vivo $[38,39]$, one might speculate about the necessity of insulin, which penetrates the blood-brain barrier $[40,41]$, in addition to palmitate co-signaling in the brain to reach the threshold in terms of hyperpolarization.

Although the intracerebroventricular application route points to a predominant role of regions and neuronal populations within the hypothalamus, it remains to be determined which brain regions are causative for the impairment of cerebrocortical activity and subsequent modulation of hepatic glucose production. For instance, it is known that neurons of hypothalamic areas project to other brain areas such as the hindbrain to regulate feeding [42], and a forebrain-hindbrain neuronal circuit was further described in mediating lipid-sensing mechanisms in the hypothalamus to inhibit glucose production [43]. In line with our findings, it is to be assumed that secondarily connected brain areas such as the hippocampus or the cerebral cortex are involved therein since effects on theta and beta activity could be revealed.

In summary, we set up a novel technique for the simultaneous assessment of brain activity by electrocorticography (ECoG) and insulin action in euglycemic-hyperinsulinemic clamps in freely moving, healthy, lean mice, combined with an intracerebroventricular application route. With this study we gained, for the first time, new insights into the time response of different intracerebroventricularly applied fatty acid species on brain activity and its contemporaneous impact on hepatic glucose production under hyperinsulinemia.

We conclude that palmitate co-signaling in the brain attenuates brain activity more when systemic insulin concentrations are increased. Such a transient reduced brain activity might be important for a contemporaneous hepatic counter-regulation to amplify peripheral insulin action. Moreover, results from human studies are somewhat limited by the fact that no mechanistic and molecular relationship between brain activity, insulin sensitivity and pancreatic beta-cell function could be revealed in which brain insulin desensitization comes along with human obesity, commonly associated with hypertriglyceridemia. As intracerebroventricular interventions or intranasal application of fatty acids cannot be undertaken in humans, the use of murine models as widely used and accepted research models of human neurological and metabolic diseases may be indicated.

Our methodological approach will be elemental for future assets that aim at better understanding the time-dependence of complex pathways involved in the bidirectional interplay between the brain and the periphery. Furthermore, the technique is highly suitable for substrate applications into the CSF as well as the arterial and venous blood pool. This will be of high value when conducting functional studies or testing pharmacological interventions for novel disease-modifying treatments in small laboratory rodents.

\section{Author Contributions}

TS and SN designed the study, performed the experiments, and analyzed data. TS and HUH wrote the manuscript. MW and TS performed surgeries and experiments. CF and IK performed statistical analysis. CF and SN notably contributed to manuscript writing. FJT, IK and MHA were involved in the manuscript revision and data interpretation. All authors have approved the final version of this manuscript for publication. TS and SN are responsible for the integrity of the data and the accuracy of the data analysis.

\section{Acknowledgments}

We gratefully acknowledge the excellent technical assistance of Juergen Schultheiß and Annett Seelig, Institute of Experimental Genetics, German Mouse Clinic, Helmholtz-Zentrum Muenchen, German Research Center for Environmental Health, Neuherberg, Germany.

\section{Funding}

This study was supported by a grant from German Center for Diabetes Research (DZD e.V.) to TS and SN. We further acknowledge support by the German Federal Ministry of Education and Research (BMBF; DLR01GI0925) to the German Center for Diabetes Research (DZD e.V.). CF, IK and FJT received funding from the European Research Council under grant agreement $n^{\circ} 259294$ (Starting grant Latent Causes) and the German Research Foundation (DFG) within the Collaborative Research Centre 1243, Subproject A17. We further acknowledge support by Open Access Publishing Fund of University of Tuebingen.

\section{Conflict of Interest}

No conflict of interest has been declared by the authors. SN is an employee of Sanofi-Aventis GmbH, Frankfurt, Germany since 2015.

\section{References}

[1] Kleinridders A, Ferris HA, Cai W et al. Insulin action in brain regulates systemic metabolism and brain function. Diabetes 2014; 63: 2232-2243

[2] Vogt MC, Bruning JC. CNS insulin signaling in the control of energy homeostasis and glucose metabolism - from embryo to old age. Trends Endocrinol Metab 2013; 24: 76-84

[3] Schwartz MW, Woods SC, Porte D Jr. et al. Central nervous system control of food intake. Nature 2000; 404: 661-671

[4] Tschritter O, Preissl H, Hennige AM et al. High cerebral insulin sensitivity is associated with loss of body fat during lifestyle intervention. Diabetologia 2012; 55: 175-182

[5] Obici S, Zhang BB, Karkanias G et al. Hypothalamic insulin signaling is required for inhibition of glucose production. Nat Med 2002; 8: 1376-1382

[6] Prodi E, Obici S. Minireview: the brain as a molecular target for diabetic therapy. Endocrinology 2006; 147: 2664-2669

[7] Heni M, Kullmann S, Preiss $\mathrm{H}$ et al. Impaired insulin action in the human brain: causes and metabolic consequences. Nat Rev Endocrinol 2015; 11: 701-711 
[8] Tschritter O, Preissl H, Hennige AM et al. The cerebrocortical response to hyperinsulinemia is reduced in overweight humans: a magnetoencephalographic study. Proc Natl Acad Sci USA 2006; 103: 12103-12108

[9] Tschritter O, Preissl H, Yokoyama Y et al. Variation in the FTO gene locus is associated with cerebrocortical insulin resistance in humans. Diabetologia 2007; 50: 2602-2603

[10] Tschritter O, Preissl H, Hennige AM et al. The insulin effect on cerebrocortical theta activity is associated with serum concentrations of saturated nonesterified Fatty acids. J Clin Endocrinol Metab 2009; 94: $4600-4607$

[11] Boden G. Role of fatty acids in the pathogenesis of insulin resistance and NIDDM. Diabetes 1997; 46: 3-10

[12] Hennige AM, Sartorius T, Lutz SZ et al. Insulin-mediated cortical activity in the slow frequency range is diminished in obese mice and promotes physical inactivity. Diabetologia 2009; 52: 2416-2424

[13] Sartorius T, Lutz SZ, Hoene M et al. Toll-like receptors 2 and 4 impair insulin-mediated brain activity by interleukin- 6 and osteopontin and alter sleep architecture. FASEB J 2012; 26: 1799-1809

[14] Veerkamp JH, Zimmerman AW. Fatty acid-binding proteins of nervous tissue. J Mol Neurosci 2001; 16: 133-142

[15] Lam TK, Pocai A, Gutierrez-Juarez R et al. Hypothalamic sensing of circulating fatty acids is required for glucose homeostasis. Nat Med 2005; 11: 320-327

[16] Obici S, Feng Z, Morgan K et al. Central administration of oleic acid inhibits glucose production and food intake. Diabetes 2002; 51: 271-275

[17] Pocai A, Lam TK, Gutierrez-Juarez R et al. Hypothalamic K(ATP) channels control hepatic glucose production. Nature 2005; 434 : 1026-1031

[18] Clore JN, Stillman JS, Li J et al. Differential effect of saturated and polyunsaturated fatty acids on hepatic glucose metabolism in humans. Am J Physiol Endocrinol Metab 2004; 287: E358-E365

[19] Xiao C, Giacca A, Carpentier A et al. Differential effects of monounsaturated, polyunsaturated and saturated fat ingestion on glucosestimulated insulin secretion, sensitivity and clearance in overweight and obese, non-diabetic humans. Diabetologia 2006; 49: 1371-1379

[20] Sartorius T, Ketterer C, Kullmann S et al. Monounsaturated fatty acids prevent the aversive effects of obesity on locomotion, brain activity, and sleep behavior. Diabetes 2012; 61: 1669-1679

[21] Boden G, Chen X, Ruiz J et al. Mechanisms of fatty acid-induced inhibition of glucose uptake. J Clin Invest 1994; 93: 2438-2446

[22] Lam TK, Carpentier A, Lewis GF et al. Mechanisms of the free fatty acid-induced increase in hepatic glucose production. Am J Physiol Endocrinol Metab 2003; 284: E863-E873

[23] Lewis GF, Vranic M, Harley P et al. Fatty acids mediate the acute extrahepatic effects of insulin on hepatic glucose production in humans. Diabetes 1997; 46: 1111-1119

[24] Rebrin K, Steil GM, Mittelman SD et al. Causal linkage between insulin suppression of lipolysis and suppression of liver glucose output in dogs. J Clin Invest 1996; 98: 741-749

[25] Wang PY, Caspi L, Lam CK et al. Upper intestinal lipids trigger a gut-brain-liver axis to regulate glucose production. Nature 2008; 452 : 1012-1016
[26] Caspi L, Wang PY, Lam TK. A balance of lipid-sensing mechanisms in the brain and liver. Cell Metab 2007; 6: 99-104

[27] Kim JK, Fillmore J], Chen Y et al. Tissue-specific overexpression of lipoprotein lipase causes tissue-specific insulin resistance. Proc Natl Acad Sci USA 2001; 98: 7522-7527

[28] Pocai A, Obici S, Schwartz G] et al. A brain-liver circuit regulates glucose homeostasis. Cell Metab 2005; 1: 53-61

[29] Ross RA, Rossetti L, Lam TK et al. Differential effects of hypothalamic long-chain fatty acid infusions on suppression of hepatic glucose production. Am J Physiol Endocrinol Metab 2010; 299: E633-E639

[30] Thaler JP, Yi CX, Schur EA et al. Obesity is associated with hypothalamic injury in rodents and humans. J Clin Invest 2012; 122: 153-162

[31] Crainiceanu CM, Staicu AM, Ray S et al. Bootstrap-based inference on the difference in the means of two correlated functional processes. Stat Med 2012; 31: 3223-3240

[32] Kaufmann S, Fuchs C, Gonik M et al. Inter-chromosomal contact networks provide insights into Mammalian chromatin organization. PLoS One 2015; 10: e0126125

[33] Holland WL, Bikman BT, Wang LP et al. Lipid-induced insulin resistance mediated by the proinflammatory receptor TLR4 requires saturated fatty acid-induced ceramide biosynthesis in mice. J Clin Invest 2011; 121: $1858-1870$

[34] Summers SA. Ceramides in insulin resistance and lipotoxicity. Prog Lipid Res 2006; 45: 42-72

[35] Boden G, She P, Mozzoli M et al. Free fatty acids produce insulin resistance and activate the proinflammatory nuclear factor-kappaB pathway in rat liver. Diabetes 2005; 54: 3458-3465

[36] Shulman Gl. Cellular mechanisms of insulin resistance. J Clin Invest 2000; 106: 171-176

[37] Lam TK, Yoshii H, Haber CA et al. Free fatty acid-induced hepatic insulin resistance: a potential role for protein kinase C-delta. Am J Physiol Endocrinol Metab 2002; 283: E682-E691

[38] Spanswick D, Smith MA, Mirshamsi S et al. Insulin activates ATP-sensitive $\mathrm{K}+$ channels in hypothalamic neurons of lean, but not obese rats. Nat Neurosci 2000; 3: 757-758

[39] Konner AC, Janoschek R, Plum L et al. Insulin action in AgRP-expressing neurons is required for suppression of hepatic glucose production. Cell Metab 2007; 5: 438-449

[40] Banks WA, Jaspan JB, Huang W et al. Transport of insulin across the blood-brain barrier: saturability at euglycemic doses of insulin. Peptides 1997; 18: 1423-1429

[41] Woods SC, Seeley RJ, Baskin DG et al. Insulin and the blood-brain barrier. Curr Pharm Des 2003; 9: 795-800

[42] Morton G], Cummings DE, Baskin DG et al. Central nervous system control of food intake and body weight. Nature 2006; 443: 289-295

[43] Lam CK, Chari M, Rutter GA et al. Hypothalamic nutrient sensing activates a forebrain-hindbrain neuronal circuit to regulate glucose production in vivo. Diabetes 2011; 60: 107-113 